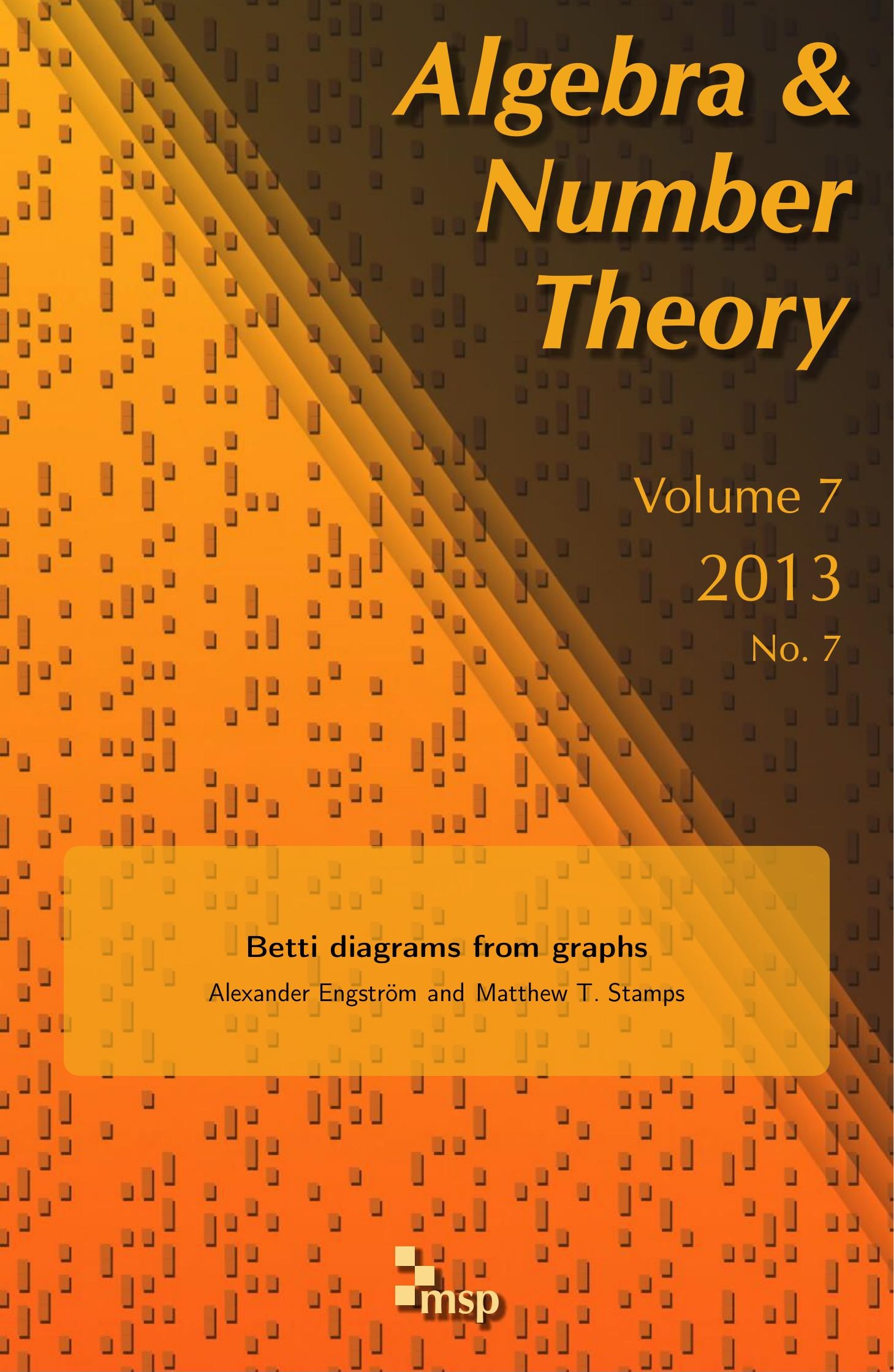




\title{
Betti diagrams from graphs
}

\author{
Alexander Engström and Matthew T. Stamps
}

\begin{abstract}
The emergence of Boij-Söderberg theory has given rise to new connections between combinatorics and commutative algebra. Herzog, Sharifan, and Varbaro recently showed that every Betti diagram of an ideal with a $k$-linear minimal resolution arises from that of the Stanley-Reisner ideal of a simplicial complex. In this paper, we extend their result for the special case of 2-linear resolutions using purely combinatorial methods. Specifically, we show bijective correspondences between Betti diagrams of ideals with 2-linear resolutions, threshold graphs, and anti-lecture-hall compositions. Moreover, we prove that any Betti diagram of a module with a 2-linear resolution is realized by a direct sum of Stanley-Reisner rings associated to threshold graphs. Our key observation is that these objects are the lattice points in a normal reflexive lattice polytope.
\end{abstract}

\section{Introduction}

A fundamental problem in commutative algebra is to characterize the coarsely graded Betti numbers of the finitely generated graded modules over a fixed polynomial ring. Originating with Hilbert in the 1890s, this task largely eluded mathematicians until 2006, when Boij and Söderberg introduced the following relaxation: Instead of trying to determine whether or not a table of nonnegative integers is the Betti diagram of a module, one should try to determine if some rational scalar of the table is the Betti diagram of a module. This shifted the viewpoint to studying rays in a rational cone and with this new geometric picture, the subject has seen a great deal of progress over the last six years. In particular, the idea led Boij and Söderberg [2008] to conjecture that every Betti diagram of a module can be decomposed in a specific and predictable way. Eisenbud and Schreyer [2009] proved this for Cohen-Macaulay modules, and Boij and Söderberg [2012] later extended that proof to the general setting.

A natural question that arises from Boij-Söderberg theory is the following: If a module is constructed from a combinatorial object, such as the edge ideal of a graph or the Stanley-Reisner ideal of a simplicial complex, can any of the combinatorial properties of that object be seen in the Boij-Söderberg decomposition of the module? Herzog, Sharifan, and Varbaro [Herzog et al. 2012] recently gave an elegant partial

MSC2010: primary 13D02; secondary 05C25.

Keywords: linear resolutions, Boij-Söderberg theory, threshold graphs. 
answer to this question for the special case of ideals with $k$-linear resolutions by showing that every Betti diagram of an ideal with a $k$-linear minimal resolution can be realized by the Stanley-Reisner ideal of a certain simplicial complex. More specifically, they prove that from the coefficients of a Boij-Söderberg decomposition of a $k$-linear Betti diagram, one obtains an $O$-sequence which, by a famous result of Eagon and Reiner along with Macaulay's theorem, yields a simplicial complex with the desired properties. Nagel and Sturgeon [2013] employ a similar approach to show that the $k$-linear Betti diagrams can be realized with hyperedge ideals of $k$-uniform Ferrers hypergraphs.

In this paper, we restrict our attention to the case of 2-linear resolutions and give an alternate characterization of the Betti diagrams of ideals with 2-linear minimal resolutions using purely combinatorial means. We show that every Betti diagram from an ideal with a 2-linear resolution is realized by a Stanley-Reisner ring constructed from a threshold graph and that this correspondence is a bijection.

Theorem 4.12. For every 2-linear ideal I in $S$, there is a unique threshold graph $T$ on $n+1$ vertices with $\beta(S / I)=\beta(\mathbb{k}[T])$.

Moreover, for any such ideal, we give an efficient algorithm for constructing its corresponding threshold graph that avoids expensive computations like Hochster's formula; rather, we can generate all such Betti diagrams recursively with affine transformations, avoiding operators such as Ext and Tor. Even more interesting, we find that these diagrams are the lattice points of a normal reflexive lattice simplex that is combinatorially equivalent to a simplex of anti-lecture-hall compositions and, from this geometric picture, we prove that any Betti diagram of a module with a 2-linear resolution arises from a direct sum of Stanley-Reisner rings constructed from threshold graphs.

Theorem 4.16. For every finitely generated, graded $S$-module $M$ with 2-linear minimal free resolution and $\beta_{0,0}(M)=m$, there is a collection of $m$ threshold graphs $\left\{T_{1}, \ldots, T_{m}\right\}$, not necessarily distinct, such that $\beta(M)=\beta\left(\mathbb{k}\left[T_{1}\right] \oplus \cdots \oplus \mathbb{k}\left[T_{m}\right]\right)$.

The paper is organized as follows: In Section 2, we give a quick review of the necessary concepts from commutative algebra and Boij-Söderberg theory. In Section 3, we interpret the main theorem of Boij-Söderberg theory in terms of linear algebra for the special case of modules with $k$-linear minimal resolutions. We prove our main theorems in Section 4 and conclude with some interesting connections to discrete geometry in Section 5.

\section{Preliminaries}

We begin with a review of the basic definitions and theorems from Boij-Söderberg theory. For a more detailed introduction, we recommend [Fløystad 2012]. 
Commutative algebra. Let $\mathbb{k}$ be a field and $S=\mathbb{k}\left[x_{1}, \ldots, x_{n}\right]$. For any finitely generated graded $S$-module $M$, let $M_{i}$ denote its graded piece of degree $i$ and let $M(d)$ denote the twisting of $M$ by $d$, that is, the module such that $M(d)_{i} \cong M_{i+d}$. A minimal graded free resolution of $M$ is an exact complex

$$
0 \leftarrow M \leftarrow F_{0} \leftarrow F_{1} \leftarrow \cdots \leftarrow F_{l},
$$

where each $F_{i}$ is a graded free $S$-module of the form

$$
\bigoplus_{j \in \mathbb{Z}} S(-j)^{\beta_{i, j}}
$$

such that the number of basis elements is minimal and each map is graded.

The value $\beta_{i, j}$ is called the $i$-th graded Betti number of degree $j$. These numbers are a refinement of the ordinary Betti numbers $\beta_{i}=\sum_{j} \beta_{i, j}$ and are independent of the choice of resolution of $M$, thus yielding an important numerical invariant of $M$. We often express the graded Betti numbers in a two-dimensional array called the Betti diagram of $M$, denoted by $\beta(M)$. Since $\beta_{i, j}=0$ whenever $i>j$, it is customary to write $\beta(M)$ such that $\beta_{i, j}$ is in position $(j-i, i)$. That is,

$$
\beta(M)=\left[\begin{array}{cccc}
\beta_{0,0} & \beta_{1,1} & \ldots & \beta_{l, l} \\
\beta_{0,1} & \beta_{1,2} & \ldots & \beta_{l, l+1} \\
\vdots & \vdots & \ddots & \vdots \\
\beta_{0, r} & \beta_{1, r+1} & \ldots & \beta_{l, l+r}
\end{array}\right] .
$$

A Betti diagram is called pure if every column has at most one nonzero entry, that is, for each $i \in\{0, \ldots, l\}, \beta_{i, j} \neq 0$ for at most one $j \in \mathbb{Z}$.

Boij-Söderberg theory. Let $\mathbb{Z}_{\mathrm{deg}}^{n+1}$ denote the set of strictly increasing nonnegative integer sequences $\boldsymbol{d}=\left(d_{0}, \ldots, d_{s}\right)$ with $s \leq n$, called degree sequences, along with the partial order given by

$$
\left(d_{0}, \ldots, d_{s}\right) \geq\left(e_{0}, \ldots, e_{t}\right)
$$

whenever $s \leq t$ and $d_{i} \geq e_{i}$ for all $i \in\{0, \ldots, s\}$. To every $\boldsymbol{d} \in \mathbb{Z}_{\mathrm{deg}}^{n+1}$, we associate a pure Betti diagram $\pi(\boldsymbol{d})$ with entries defined as follows:

$$
\pi_{i, j}(\boldsymbol{d})=\left\{\begin{array}{cl}
\prod_{k \neq 0, i}\left|\frac{d_{k}-d_{0}}{d_{k}-d_{i}}\right| & i \geq 0, j=d_{i}, \\
0 & \text { otherwise. }
\end{array}\right.
$$

The main theorem of Boij-Söderberg theory states that the Betti diagram of any graded $S$-module can be written as a positive rational combination of $\pi(\boldsymbol{d})$ 's. It was originally conjectured by Boij and Söderberg [2008], proven for Cohen-Macaulay modules by Eisenbud and Schreyer [2009], and then generalized thus: 
Theorem 2.1 [Boij and Söderberg 2012]. For every graded S-module $M$, there exists a vector $c \in \mathbb{Q}_{\geq 0}^{p}$ and a chain of degree sequences $\boldsymbol{d}^{1}<\boldsymbol{d}^{2}<\cdots<\boldsymbol{d}^{p}$ in $\mathbb{Z}_{\mathrm{deg}}^{n+1}$ such that

$$
\beta(M)=c_{1} \pi\left(\boldsymbol{d}^{1}\right)+\cdots+c_{p} \pi\left(\boldsymbol{d}^{p}\right) .
$$

The combination in Theorem 2.1 is called a Boij-Söderberg decomposition of $M$ and the entries of $c$ are called Boij-Söderberg coefficients. This decomposition is not unique in general, but there is a simple algorithm for computing a set of coefficients that satisfy the theorem, see [Fløystad 2012].

\section{Betti diagrams of 2-linear resolutions}

An ideal $I$ in $S$ is called $k$-linear if $\beta_{i, j}(I)=0$ whenever $j-i \neq k-1$. If $I$ is 2-linear, then the Betti diagram of $M=S / I$ looks like

$$
\beta(M)=\left[\begin{array}{cccccc}
1 & \cdot & \cdot & \cdot & \cdots & \cdot \\
\cdot & \beta_{1} & \beta_{2} & \beta_{3} & \cdots & \beta_{s}
\end{array}\right]
$$

for some $s \leq n$. Our aim is to translate the statement of Theorem 2.1, for $S$-modules with 2-linear resolutions, into linear algebraic terms. For this, it will be convenient to consider the reduced Betti vector $\omega(M)=\left[\beta_{1}, \ldots, \beta_{s}\right]$ in place of $\beta(M)$.

If $M$ is a 2-linear $S$-module, then every $\boldsymbol{d}^{l}$ in Theorem 2.1 is of the form $(0,2, \ldots, l+1)$. So, let $\pi^{l}=\pi\left(\boldsymbol{d}^{l}\right), \omega^{l}$ be the reduced Betti vector corresponding to $\pi^{l}$, and $\Omega$ be the lower-diagonal $n \times n$ matrix whose $l$-th row is $\omega^{l}$. We leave it to the reader to verify the following:

Lemma 3.1. The matrix $\Omega$ is invertible and has $i j$-entry $\omega_{j}^{i}=j\left(\begin{array}{c}i+1 \\ j+1\end{array}\right)$. Moreover, the ij-entry of $\Omega^{-1}$ is $(-1)^{i-j} \frac{1}{i}\left(\begin{array}{c}i+1 \\ j+1\end{array}\right)$.

Since any subset of row vectors in $\Omega$ forms a chain in $\mathbb{Z}_{\text {deg }}^{n+1}$, we can replace the vector $c \in \mathbb{Q}_{>0}^{p}$ in Theorem 2.1 with a vector $c \in \mathbb{Q}_{\geq 0}^{n}$ such that $\sum_{i} c_{i}=\beta_{0,0}(M)$.

Theorem 3.2. For every 2-linear (graded) $S$-module $M$ with $\beta_{0,0}(M)=m$,

$$
\beta(M)=c_{1} \pi^{1}+\cdots+c_{n} \pi^{n},
$$

where $c=\omega(M) \Omega^{-1} \in \mathbb{Q}_{\geq 0}^{n}$ and $\sum_{i} c_{i}=m$.

Remark 3.3. When $\beta_{0,0}(M)=1$, Theorem 3.2 asserts that $\omega(M)$ is a lattice point in the $(n-1)$-dimensional simplex spanned by row vectors of $\Omega$.

We conclude this section with some classic examples of 2-linear ideals that arise from graph theory. A graph $G$ consists of a finite set $V(G)$, called the vertex set, and a subset $E(G)$ of $\left(\begin{array}{c}V(G) \\ 2\end{array}\right)$, called the edge set. To simplify notation, we write $u v$ instead of $\{u, v\}$ for each edge in $G$. For any subset of vertices $W \subset V(G)$, the induced subgraph $G[W]$ is the graph with vertex set $W$ and edge set $E(G) \cap\left(\begin{array}{c}W \\ 2\end{array}\right)$. 
If $W=V(G) \backslash S$ for some $S \subseteq V(G)$, we write $G \backslash S$ instead of $G[W]$. A subgraph $C$ of the form $V(C)=\left\{v_{1}, \ldots, v_{l}\right\}$ and $E(C)=\left\{v_{i} v_{i+1} \mid 1 \leq i<l\right\} \cup\left\{v_{1} v_{l}\right\}$ is called a cycle of length $l$. We say $G$ is chordal if it has no induced cycles of length greater than three or, equivalently, if $E(C) \subsetneq E(G[C])$ for every cycle of length greater than three. The elements of $E(G[C]) \backslash E(C)$ are called chords. Chordal graphs have many interesting properties that are actively studied in graph theory. For a thorough introduction to graph theory, we recommend [Diestel 2010].

Given a graph $G$ with vertex set $[n+1]=\{1, \ldots, n+1\}$, where $n$ is the number of indeterminates in $S$, let $R=\mathbb{k}\left[x_{1}, \ldots, x_{n+1}\right]$, let

$$
I^{c}(G)=\left\langle x_{i} x_{j} \mid i j \notin E(G)\right\rangle \subseteq R
$$

be the ideal generated by the monomials corresponding to nonedges in $G$, and let $\mathbb{k}[G]$ be the quotient $R / I^{c}(G)$. The knowledgeable reader may observe that $I^{c}(G)$ is the edge ideal of the complement of $G$ and $\mathbb{k}[G]$ is the Stanley-Reisner ring of the clique complex of $G$. The following theorem was first proved by Fröberg [1990] and then by Dochtermann and Engström [2009], using topological combinatorics.

Theorem 3.4. A graph $G$ is chordal if and only if $I^{c}(G)$ is 2-linear. Whenever this is the case,

$$
\beta_{i, j}(\mathbb{k}[G])=\sum_{W \in\left(\begin{array}{c}
V(G) \\
j
\end{array}\right)}(-1+\# \text { components of } G[W])
$$

for $i=j-1 \geq 1$.

Example 3.5. If $G$ consists of $n+1$ isolated vertices, then the $\left(\begin{array}{c}n+1 \\ i+1\end{array}\right)$ induced subgraphs of $G$ with $i+1$ vertices each have $i+1$ connected components. Thus, $\beta_{i, i+1}(\mathbb{k}[G])=i\left(\begin{array}{c}n+1 \\ i+1\end{array}\right)$ for each $i \geq 1$.

Example 3.6. If $G$ consists of a complete graph on $n$ vertices plus an isolated vertex $v$, then the $\left(\begin{array}{l}n \\ i\end{array}\right)$ induced subgraphs of $G$ with $i+1$ vertices that contain $v$ each have two connected components and the remaining induced subgraphs of $G$ (with $i+1$ vertices) are connected. Thus, $\beta_{i, i+1}(\mathbb{k}[G])=\left(\begin{array}{l}n \\ i\end{array}\right)$ for each $i \geq 1$.

Remark 3.7. If we apply Theorems 3.2 and 3.4 to $\mathbb{k}[G]$ for some chordal graph $G$, we get a formula that takes the number of connected components of induced subgraphs of $G$ as input and yields a vector $c \in \mathbb{Q}_{\geq 0}^{n}$, namely $\omega(\mathbb{k}[G]) \Omega^{-1}$, whose entries sum to 1 . It is natural to ask what this formula says if $G$ is not chordal. If the entries of $c$ fail to be nonnegative or sum to 1 , then we get a certificate that $G$ is not chordal. Since measuring how far a graph is from being chordal is nontrivial from the viewpoint of complexity, one is inclined to ask if this procedure characterizes chordal graphs.

Alas, this turns out to not be the case - there are nonchordal graphs that yield admissible c's - but these false chordal graphs seem to be few. Examples of false 

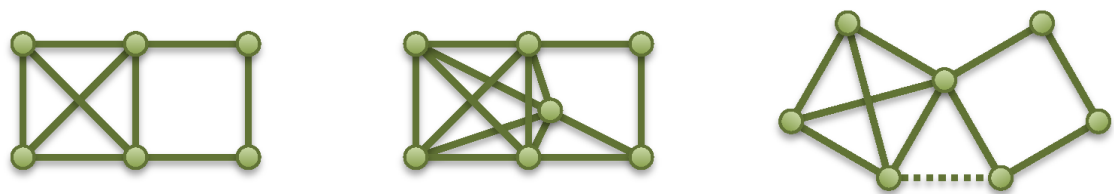

Figure 1. The single false chordal graph on six vertices along with two examples on seven vertices.

chordal graphs on six and seven vertices are illustrated in Figure 1. All other false chordal graphs on seven vertices arise from expanding a (possibly empty) clique of the six-vertex graph or coning over the whole six-vertex graph. We offer some computer-generated statistics on the size of each class of graphs for a given number of vertices:

\begin{tabular}{r|rrrrrrr} 
& 1 & 2 & 3 & 4 & 5 & 6 & 7 \\
\hline Chordal & 1 & 2 & 4 & 10 & 27 & 94 & 393 \\
False chordal & 0 & 0 & 0 & 0 & 0 & 1 & 15 \\
Not chordal & 0 & 0 & 0 & 1 & 7 & 62 & 651
\end{tabular}

\section{Betti diagrams from graphs}

In this section, we study the Betti diagrams corresponding to a special class of chordal graphs called threshold graphs. We show that threshold graphs on a fixed vertex set have distinct Betti diagrams, that every Betti diagram of a chordal graph is that of a threshold graph on the same number of vertices, that every Betti diagram of an $S$-algebra with a 2-linear resolution is that of a threshold graph on $n+1$ vertices, and that every Betti diagram of an $S$-module with a 2-linear resolution is that of a direct sum of Stanley-Reisner rings constructed from threshold graphs on $n+1$ vertices, where $n$ is the number of indeterminates in $S$.

Betti diagrams from threshold graphs. In a graph $G$, two vertices are said to be adjacent if they are contained in an edge of $G$. A vertex adjacent to no others is called isolated and a vertex adjacent to all others is called dominating. For every graph $G$ on $n$ vertices, let $G_{*}$ be the graph on $n+1$ vertices obtained by adding an isolated vertex to $G$ and, similarly, let $G^{*}$ be the graph obtained by adding a dominating vertex to $G$. A graph $G$ is called threshold if it can be constructed from a single vertex and a sequence of the operations * and ${ }_{*}$. It is well known that if $G$ is chordal, then so are $G_{*}$ and $G^{*}$, and thus, all threshold graphs are chordal. We refer to Mahadev and Peled [1995] for a survey that includes the following lemma:

Lemma 4.1. There are $2^{n}$ threshold graphs on $n+1$ vertices. Moreover, every threshold graph is determined by a unique sequence of ${ }^{*}$ and ${ }_{*}$ operations. 
The Betti diagram of a threshold graph can be constructed recursively in a similar manner to the graph itself. As such, we can quickly calculate the Betti diagram of a threshold graph without the computations in Theorem 3.4.

Proposition 4.2. If $G$ is a chordal graph on $n$ vertices, then

$$
\omega\left(\mathbb{k}\left[G^{*}\right]\right)=[\omega(\mathbb{k}[G]) \mid 0] \quad \text { and } \quad \omega\left(\mathbb{k}\left[G_{*}\right]\right)=\omega(\mathbb{k}[G]) \Lambda+\eta_{n},
$$

where $\Lambda$ is the $(n-1) \times n$-matrix whose $(i, j)$ position is 1 if $i=j$ or $j-1$ and 0 otherwise, and $\eta_{n}$ is the vector whose $i$-th entry is $\left(\begin{array}{c}n \\ i\end{array}\right)$.

Proof. This is a simple application of Theorem 3.4. For the first part, any subset of vertices containing the dominating vertex in $G^{*}$ spans a connected graph and therefore, the only nonzero parts of $\omega\left(\mathbb{k}\left[G^{*}\right]\right)$ come from $\omega(\mathbb{k}[G])$. For the second part, we consider whether or not a subset of vertices in $G_{*}$ contains the isolated vertex $v$ : The induced subgraphs that do not contain $v$ contribute $[\omega(\mathbb{k}[G]) \mid 0]$ to $\omega\left(\mathbb{k}\left[G_{*}\right]\right)$ while those that $d o$ contain $v$ contribute $[0 \mid \omega(\mathbb{k}[G])]+\eta_{n}$.

As a corollary, we find that distinct threshold graphs on a fixed number of vertices have distinct Betti diagrams.

Corollary 4.3. If $T$ and $T^{\prime}$ are threshold graphs on the same number of vertices and $\omega(\mathbb{k}[T])=\omega\left(\mathbb{k}\left[T^{\prime}\right]\right)$, then $T \cong T^{\prime}$.

Proof. For any chordal graph $G$ on $k$ vertices, $\omega_{k+1}\left(\mathbb{k}\left[G_{*}\right]\right) \neq \omega_{k+1}\left(\mathbb{k}\left[G^{*}\right]\right)=0$ by Proposition 4.2. Therefore, since distinct threshold graphs have distinct sequences of $*$ and * (Lemma 4.1), they must also have distinct Betti diagrams.

Betti diagrams from chordal graphs. Next, we show that every Betti diagram from a chordal graph arises as the Betti diagram of a threshold graph on the same number of vertices. Moreover, for a given chordal graph, we present an efficient algorithm for constructing its "threshold representative".

Let $\sim_{\beta}$ be the equivalence relation for graphs on $[n+1]$ defined by

$$
G \sim_{\beta} H \text { if and only if } \beta(\mathbb{k}[G])=\beta(\mathbb{k}[H])
$$

and let $[G]_{\beta}$ denote the equivalence class of $G$ with respect to $\sim_{\beta}$. For a chordal graph $G$ on $n+1$ vertices, a threshold graph $T$ (on $n+1$ vertices) is called a threshold representative of $G$ if $T \in[G]_{\beta}$. The next theorem follows from the notion of algebraic shifting and can be pieced together from results in [Goodarzi and Yassemi 2012; Klivans 2007; Woodroofe 2011], but we offer a purely graph-theoretic proof instead.

Theorem 4.4. Every chordal graph $G$ has a unique threshold representative T. 

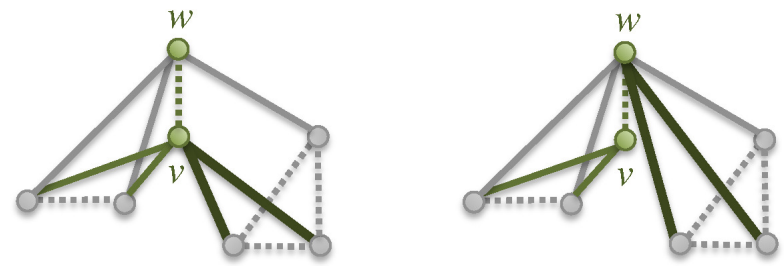

Figure 2. A comparison of a graph $G$ (left) with $G_{v \rightarrow w}$ (right).

We proceed with some new machinery. For a graph $G$ with $v, w \in V(G)$, we define a new graph $G_{v \rightarrow w}$ on $V(G)$ with

$$
E\left(G_{v \rightarrow w}\right):=(E(G) \backslash\{u v \mid u \in N(v ; w)\}) \cup\{u w \mid u \in N(v ; w)\},
$$

where $N(x)=\{y \in V(G) \mid x y \in E(G)\}$ is the neighborhood of a vertex $x$ and $N(v ; w)=N(v) \backslash(\{w\} \cup N(w))$. See Figure 2.

Lemma 4.5. Let $G$ be a chordal graph.

(1) If $G$ is connected with $v w \in E(G)$, then $G_{v \rightarrow w}$ is chordal.

(2) If $G$ is disconnected with $v, w \in V(G)$ in separate components, then $G_{v \rightarrow w}$ is chordal.

Proof. For each part, we suppose $C$ is a cycle with length $l \geq 4$ in $G^{\prime}=G_{v \rightarrow w}$ and show that $C$ has a chord in $G^{\prime}$.

In (1), if $w \notin V(C)$, then $C \subseteq G$ since the only new edges of $G^{\prime}$ contain $w$ and therefore $C$ has at least one chord in $G$. If every chord of $C$ in $G$ is removed in $G^{\prime}$, then they must each contain $v$ and thus $G[V(C \backslash v) \cup w]$ is an induced cycle, which is a contradiction. If $w \in V(C), v \notin V(C)$, and $C$ does not have a chord in $G^{\prime}$, then $G[V(C) \cup v]$ is an induced cycle since $N(v) \subseteq N(w)$ in $G^{\prime}$, another contradiction. If $v, w \in V(C)$, then $v w \in E(C)$ and $x w$ is a chord of $C$ in $G^{\prime}$, where $x$ is the other neighbor of $v$ in $C$, since $N(v) \subseteq N(w)$ in $G^{\prime}$.

In (2), if $w \notin V(C)$, then $C$ contains a chord in $G \backslash w=G^{\prime} \backslash w \subseteq G^{\prime}$. So suppose $w \in V(C)$ and $C$ has no chord in $G^{\prime}$. Then $G[V(C \backslash w)]$ is contained in the connected component of either $v$ or $w$ in $G$. If the former is true, then $G[V(C \backslash w) \cup v]$ is an induced cycle and if the latter is true, then $C$ itself is an induced cycle in $G$, both of which are contradictions.

For a graph $H$ with $W \subseteq V(H)$, let $\kappa_{H}(W)$ denote the number of connected components in $H[W]$.

Lemma 4.6. Let $G$ be a chordal graph.

(1) If $G$ is connected with $v w \in E(G)$, then $G_{v \rightarrow w} \in[G]_{\beta}$.

(2) If $G$ is disconnected with $v, w \in V(G)$ in separate components, then $G_{v \rightarrow w}$ is in $[G]_{\beta}$. 
Proof. This is a straightforward application of Theorem 3.4 after we make the following calculations. For each part, let $G^{\prime}=G_{v \rightarrow w}$ and $W \subseteq V(G)$.

In (1), if $v, w \notin W$, then $\kappa_{G}(W)=\kappa_{G^{\prime}}(W)$ since $G \backslash\{v, w\}=G^{\prime} \backslash\{v, w\}$ and if $v, w \in W$, then $\kappa_{G}(W)=\kappa_{G^{\prime}}(W)$ because the component in $G[W]$ containing $v$ and $w$ spans the same set of vertices as that of $G^{\prime}[W]$. For the remaining subsets of $V(G)$, we prove that $\kappa_{G}(W \cup v)+\kappa_{G}(W \cup w)=\kappa_{G^{\prime}}(W \cup v)+\kappa_{G^{\prime}}(W \cup w)$ for every $W \subseteq V(G) \backslash\{v, w\}$. Let $m_{\circ}(W), m_{w}(W)$, and $m_{v}(W)$ denote the number of connected components of $G[W]$ that do not contain any elements of $N(v) \cup N(w)$, $N(v) \backslash N(w)$, and $N(w) \backslash N(v)$, respectively. It is straightforward to check that $\kappa_{G}(W \cup v)=1+m_{\circ}(W)+m_{w}(W), \kappa_{G}(W \cup w)=1+m_{\circ}(W)+m_{v}(W), \kappa_{G^{\prime}}(W \cup v)=$ $1+m_{\circ}(W)+m_{v}(W)+m_{w}(W)$, and $\kappa_{G^{\prime}}(W \cup w)=1+m_{\circ}(W)$.

In (2), we record the difference between $\kappa_{G}(W)$ and $\kappa_{G^{\prime}}(W)$. If $v, w \notin W$, then $\kappa_{G}(W)=\kappa_{G^{\prime}}(W)$ since $G \backslash\{v, w\}=G^{\prime} \backslash\{v, w\}$. If $v, w \in W$, then $\kappa_{G}(W)=\kappa_{G^{\prime}}(W)$ because every vertex in the component of $v$ in $G[W]$ gets moved to the component of $w$ in $G^{\prime}[W]$. If $v \in W$ and $w \notin W$, then $\kappa_{G}(W)=\kappa_{G^{\prime}}(W)-1$. If $w \in W$ and $v \notin W$, then $\kappa_{G}(W)=\kappa_{G^{\prime}}(W)+1$.

Proof of Theorem 4.4. We induct on $|V(G)|$. Let $G$ be a chordal graph on $n$ vertices and fix a vertex $v \in V(G)$. We will apply the operations ${ }_{v \rightarrow w}$ or $w \rightarrow v$ to $G$ to a get a graph where $v$ is either dominating or isolated.

If $G$ is connected and $v$ is not dominating, then for any vertex $u \in G$ with $d(u, v)=2$, let $w \in N(v) \cap N(u)$ and replace $G$ with $G_{w \rightarrow v}$. Repeat this until $v$ is a dominating vertex, that is, there are no more elements $u$ with $d(v, u)=2$. The process terminates since $G$ is finite and connected. By Lemma 4.5 , the graph $G$ is chordal at every step and by Lemma 4.6, its Betti diagram stays fixed. Since $v$ is dominating and $G \backslash v$ is chordal (being an induced subgraph of a chordal graph), $\beta(\mathbb{k}[G])=\beta(\mathbb{k}[G \backslash v])$. So, by induction, there is a unique (up to isomorphism) threshold graph $T$ such that $\beta\left(\mathbb{k}\left[T^{*}\right]\right)=\beta(\mathbb{k}[T])=\beta(\mathbb{k}[G \backslash v])=\beta(\mathbb{k}[G])$.

If $G$ is disconnected, let $w \in V(G)$ be in a separate component in $G$ from $v$. By Lemmas 4.5 and 4.6, $G_{v \rightarrow w}$ is chordal and $\beta(\mathbb{k}[G])=\beta\left(\mathbb{k}\left[G_{v \rightarrow w}\right]\right)$; by induction, there exists a unique (up to isomorphism) threshold graph $T \in\left[G_{v \rightarrow w} \backslash v\right]_{\beta}$. Thus, $T_{*}=T \cup\{\alpha\} \in[G]_{\beta}$ and $\beta\left(\mathbb{k}\left[T_{*}\right]\right)=\beta(\mathbb{k}[G])$.

Remark 4.7. The algorithm presented in the proof of Theorem 4.4 is fast. A crude analysis of the complexity is as follows: For each vertex of $G$, we decompose $G$ into its connected components which takes $O(|V(G)|+|E(G)|)$ and then we repeatedly apply the operations $v \rightarrow w$ or $w \rightarrow v$; by amortized analysis, this takes only $O(|E(G)|)$ since each edge is moved at most once. Thus, the total complexity is $O(|V(G)|(|V(G)|+|E(G)|)) \approx O\left(|V(G)|^{3}\right)$. The authors suspect that a more thorough analysis would yield a complexity of $O\left(|V(G)|^{2}\right)$, which is the best one could hope for with this problem. 
As simple corollaries of Theorem 4.4, we recover two special classes of graphs that are invariant under $\beta$.

Corollary 4.8. If $G$ is a tree on $n+1$ vertices, then $\beta_{i, i+1}(\mathbb{k}[G])=i\left(\begin{array}{c}n \\ i+1\end{array}\right)$.

Proof. Since $G$ has exactly $n$ edges and $v \rightarrow w$ preserves the number of edges in $G$, the procedure outlined in the proof of Theorem 4.4 yields a threshold representative $T$ of $G$ that is a star on $n+1$ vertices, that is, a single dominating vertex $v$ and no other edges. Therefore, $T \backslash v$ consists of $n$ isolated points and, by Proposition 4.2 and Example 3.5, $\beta_{i, i+1}(\mathbb{k}[G])=\beta_{i, i+1}(\mathbb{k}[T])=\beta_{i, i+1}(\mathbb{k}[T \backslash v])=(i)\left(\begin{array}{c}n \\ i+1\end{array}\right)$.

The graph from a triangulation of a polygon is called maximally outerplanar.

Corollary 4.9. If $G$ is a maximal outerplanar graph on $n+1$ vertices, then $\beta_{i, i+1}(\mathbb{k}[G])=i\left(\begin{array}{c}n-1 \\ i+1\end{array}\right)$.

Proof. By Theorem 4.4, the threshold representative $T$ of $G$ consists of a dominating vertex $v$ and a path on $V(T) \backslash v$. In particular, $T \backslash v$ is a tree on $n$ vertices. The result now follows from Proposition 4.2 and Corollary 4.8.

Betti diagrams of algebras and modules. Here we present the main results of the paper - that every Betti diagram from a 2-linear ideal in $S$ arises from a StanleyReisner ring of a threshold graph on $n+1$ vertices and that every Betti diagram from an $S$-module with a 2-linear resolution arises from a direct sum of Stanley-Reisner rings constructed from threshold graphs on $n+1$ vertices.

To begin, we establish bijections between the set of threshold graphs on $n+1$ vertices, the set of Betti diagrams from 2-linear ideals in $S$, and the set of antilecture-hall compositions of length $n$ bounded above by 1 . An integer sequence $\lambda=\left(\lambda_{1}, \lambda_{2}, \ldots, \lambda_{n}\right)$ of the form

$$
t \geq \frac{\lambda_{1}}{1} \geq \frac{\lambda_{2}}{2} \geq \cdots \geq \frac{\lambda_{n}}{n} \geq 0
$$

is called anti-lecture-hall composition of length $n$ bounded above by $t$. These sequences were introduced in [Corteel and Savage 2003] and are a well-studied variation of the lecture hall partitions in [Bousquet-Mélou and Eriksson 1997a; 1997b]. For our purposes, we only need this result of Corteel, Lee, and Savage:

Theorem 4.10 ([Corteel et al. 2005]). There are $(t+1)^{n}$ anti-lecture-hall compositions of length $n$ bounded above by $t$.

We remark that $\mathbb{k}[G]=R$ if $G$ is the complete graph on $n+1$ vertices, so we shall ignore that graph for the rest of the paper.

Proposition 4.11. The set of noncomplete threshold graphs on $n+1$ vertices, the set of Betti diagrams of quotients of $S$ by 2-linear ideals, and the set of anti-lecture-hall compositions of length $n$ with $\lambda_{1}=1$ are in bijective correspondence. 
Proof. By Lemma 4.1 and Corollary 4.3, there are $2^{n}-1$ noncomplete threshold graphs on $n+1$ vertices, each of which corresponds to a distinct Betti diagram. It suffices to show that the Betti diagrams of quotients of $S$ by 2-linear ideals inject into the anti-lecture-hall compositions of length $n$ with $\lambda_{1}=1$, since by Theorem 4.10 , there are exactly $2^{n}-1$ of them.

Let $I$ be a 2-linear ideal in $S$ and let $\Psi$ be the unimodular matrix with $i j$-entry equal to $\left(\begin{array}{c}i-1 \\ j-1\end{array}\right)$. Then there exists $\lambda=\left[\lambda_{1}, \ldots, \lambda_{n}\right] \in \mathbb{Z}^{n}$ such that $\omega(S / I)=\lambda \Psi$. By Theorem 3.2, we have

$$
\lambda \Psi \Omega^{-1}=\left[c_{1}, \ldots, c_{n}\right] \in \mathbb{Q}_{\geq 0}^{n}
$$

with $\sum_{i=1}^{n} c_{i}=1$. We leave it to the reader to verify that $\Psi \cdot \Omega^{-1}$ has $i j$-entry $1 / i$ if $i=j,-1 / i$ if $i=j+1$, and 0 otherwise. Thus, $c_{i}=\lambda_{i} / i-\lambda_{i+1} /(i+1)$ for all $i \in[n-1]$ and $c_{n}=\lambda_{n} / n$. In particular, we get

$$
1=\sum_{i=1}^{n} c_{i}=\frac{\lambda_{1}}{1} \geq \frac{\lambda_{2}}{2} \geq \cdots \geq \frac{\lambda_{n}}{n}=c_{n} \geq 0
$$

and hence, $\lambda$ is an anti-lecture-hall composition with $\lambda_{1}=1$.

The first part of our main theorem is a simple corollary of Proposition 4.11. In particular, it asserts that the injection in Proposition 4.2 is in fact a bijection.

Theorem 4.12 (Main Theorem, Part 1). For every 2-linear ideal I in S, there is a unique threshold graph $T$ on $n+1$ vertices with $\beta(S / I)=\beta(\mathbb{k}[T])$.

Remark 4.13. For a given 2-linear ideal $I$ in $S$, it is easy to construct the graph $T$ realizing its Betti diagram.

Example 4.14. To illustrate Theorem 4.12 at work, consider the ideal

$$
I=\left\langle x_{1}^{2}, x_{1} x_{2}, x_{1} x_{3}, x_{1} x_{4}, x_{2}^{2}, x_{1} x_{5}+x_{2} x_{4}, x_{4}^{2}\right\rangle \subseteq S=\mathbb{k}\left[x_{1}, \ldots, x_{5}\right] .
$$

Then

$$
\beta(S / I)=\left[\begin{array}{cccccc}
1 & \cdot & \cdot & \cdot & \cdot & \cdot \\
\cdot & 7 & 11 & 6 & 1 & 0
\end{array}\right]
$$

In order to find a threshold graph $T$ on six vertices whose Betti diagram is $\beta(S / I)$, we sequentially apply the inverses of the affine transformations in Proposition 4.2 depending on whether or not the sequences end in 0 . (We leave it to the reader to verify that the inverse of $\Lambda$ in Proposition 4.2 is the $n \times(n-1)$-matrix whose $(i, j)$ position is $(-1)^{i+j}$ if $i \leq j$ and 0 otherwise.)

$$
[7,11,6,1,0] \stackrel{-^{*}}{\longrightarrow}[7,11,6,1] \stackrel{-_{*}}{\longrightarrow}[3,2,0] \stackrel{-^{*}}{\longrightarrow}[3,2] \stackrel{-_{*}}{\longrightarrow}[1] \stackrel{-_{*}}{\longrightarrow}[0]
$$




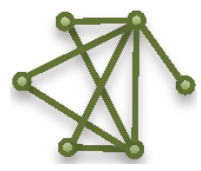

Figure 3. The threshold graph $T$ on six vertices with $\omega(\mathbb{k}[T])=[7,11,6,1,0]$.

From this, we see that $\beta(S / I)=\beta(\mathbb{k}[T])$, where $T$ is the threshold graph with sequence ${ }_{* *}{ }^{*} *$ drawn in Figure 3 .

For the rest of the paper, we take a more geometric approach. Specifically, we make use of the fact (Remark 3.3) that the reduced Betti vectors of these diagrams are lattice points in the $(n-1)$-dimensional lattice simplex $P_{n}$ spanned by the row vectors of $\Omega$. Illustrations of $P_{1}$ through $P_{4}$, labeled by reduced Betti vectors, Boij-Söderberg coefficients, truncated coordinates (see Section 5), and corresponding chordal graphs are shown in Figures 4 and 5, with the threshold graphs colored dark green. Notice that each $P_{n}$ contains two copies of $P_{n-1}$, colored blue and red, corresponding to the first and second equations, respectively, in (1) (see Proposition 4.2).

We continue with some standard definitions from discrete geometry. The integer points $\mathbb{Z}^{d} \subseteq \mathbb{R}^{d}$ form a lattice. The integer points of a polytope are its lattice points
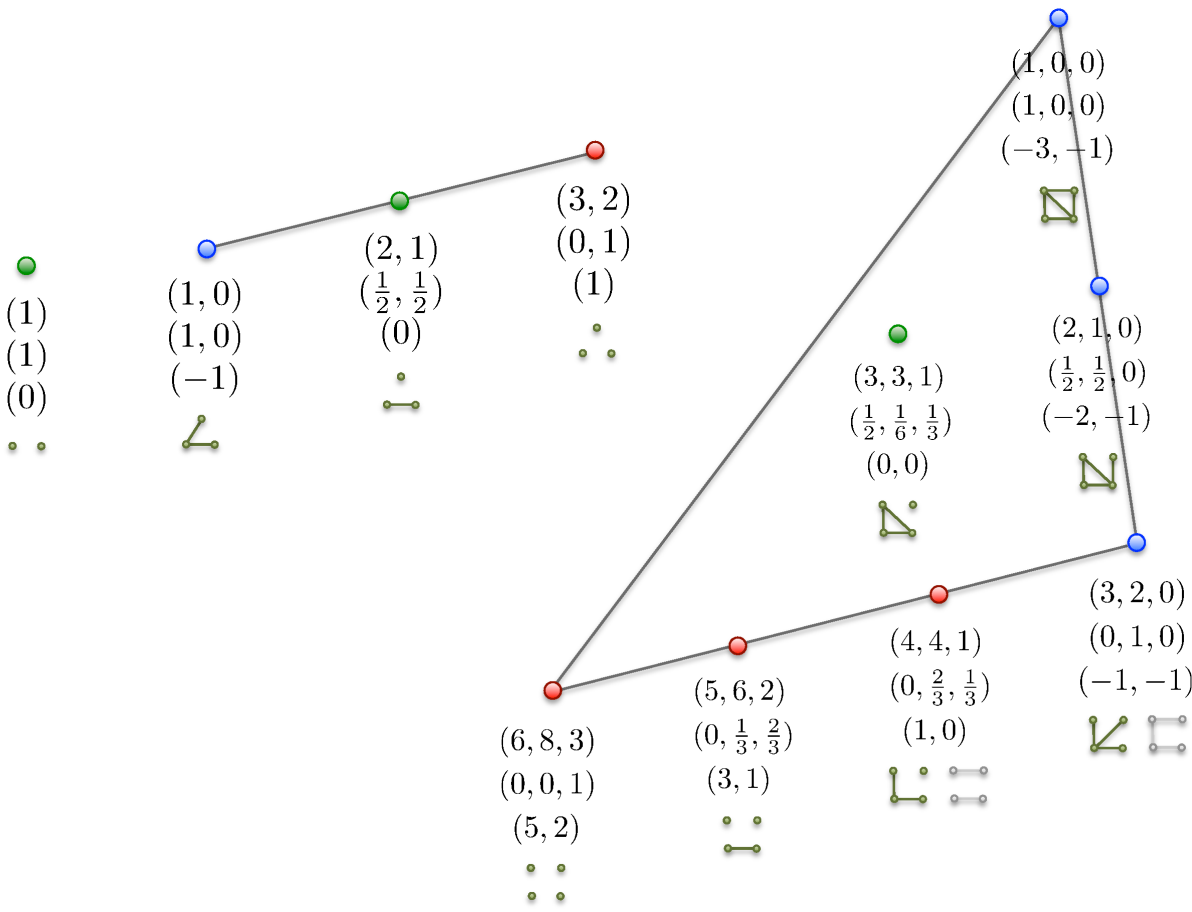

Figure 4. The lattice polytopes $P_{1}$ (left), $P_{2}$ (middle), and $P_{3}$ (right). 


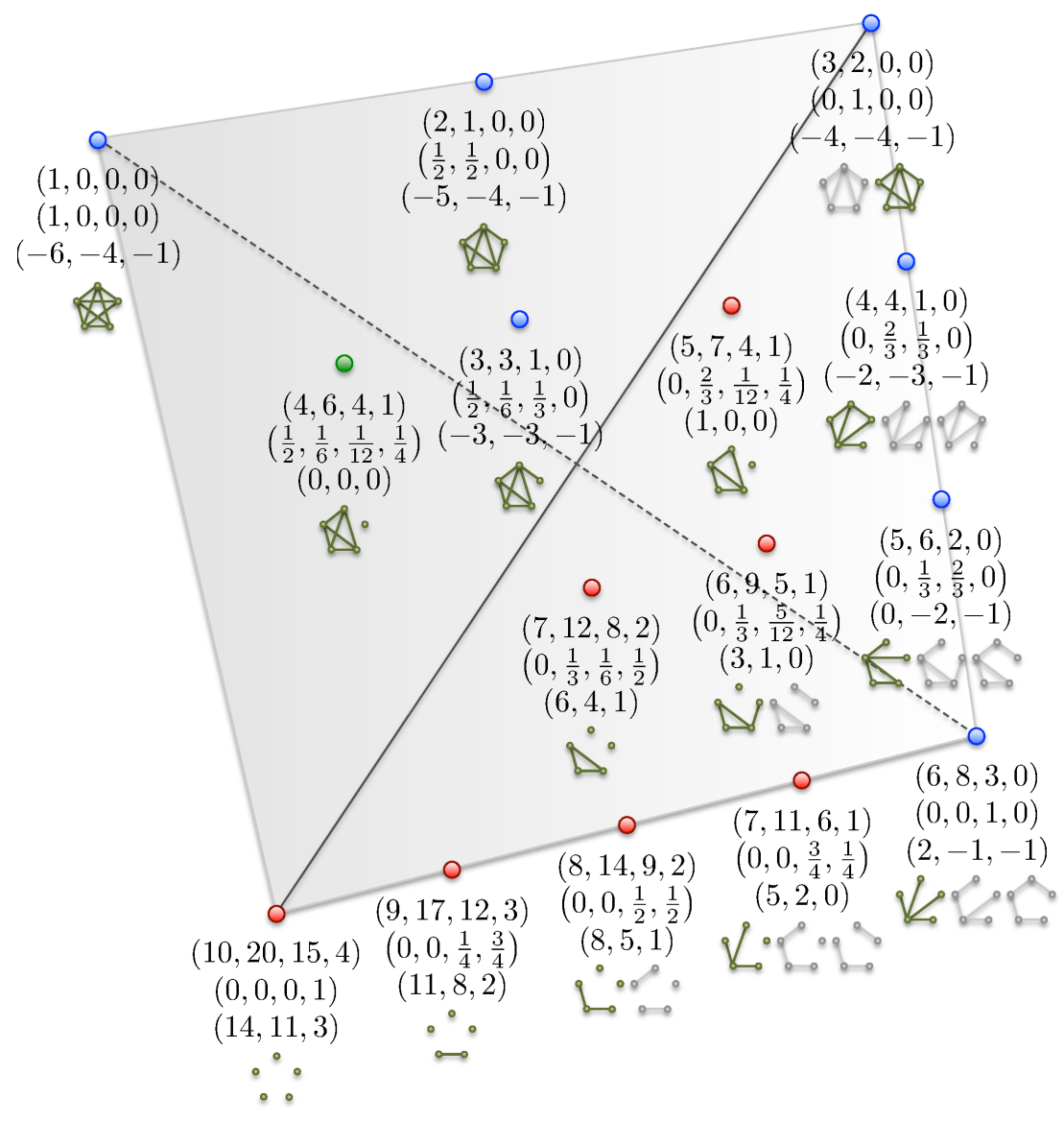

Figure 5. The lattice polytope $P_{4}$.

and a polytope is called a lattice polytope if all its vertices are lattice points. For a polytope $P$ with vertices $\left\{v_{1}, \ldots, v_{s}\right\}$ and $t \in \mathbb{N}$, let $t P$ denote the $t$-th dilation of $P$, that is, the polytope attained by taking the convex hull of the points $\left\{t \cdot v_{1}, \ldots, t \cdot v_{s}\right\}$, let $S_{P} \subseteq \mathbb{Z}^{d+1}$ denote the semigroup generated by $\left\{\left[1, p_{1}, \ldots, p_{d}\right]:\left(p_{1}, \ldots, p_{d}\right) \in\right.$ $\left.P \cap \mathbb{Z}^{d}\right\}$, and let $\operatorname{gp}\left(S_{P}\right)$ be the smallest group containing $S_{P}$, that is the group of differences in $S_{P}$. We say $P$ is normal if $x \in \operatorname{gp}\left(S_{P}\right)$ such that $s \cdot x \in S_{P}$ for some $s \in \mathbb{N}$ implies that $x \in S_{P}$. We refer to [Barvinok 2002; Bruns et al. 1997] for questions on lattice polytopes.

Proposition 4.15. The lattice simplex $P_{n}$ is normal for each $n \in \mathbb{N}$.

Proof. It is straightforward to check that the anti-lecture-hall compositions of length $n$ bounded above by 1 are the lattice points of the $n$-dimensional lattice simplex spanned by $(0, \ldots, 0)$ and the compositions $\lambda^{l}=(1,2, \ldots, l, 0, \ldots, 0)$ for $l \in[n]$. Let $Q_{n}$ be the facet spanned by the $\lambda^{l}$. Since normality is preserved 
under unimodular transformations, we prove that $Q_{n}$ is normal and apply $\Psi$ from the proof of Proposition 4.11.

To begin, we must truncate the coordinates of $Q_{n}$ since it is an $(n-1)$-dimensional simplex. Removing the first coordinate yields the simplex with vertices $(0, \ldots, 0)$ and $(2,3, \ldots, l, 0, \ldots, 0)$ for $l \in[n]$. Then $S_{Q_{n}}$ is the set of all anti-lecture-hall compositions and $\operatorname{gp}\left(S_{Q_{n}}\right)=\mathbb{Z}^{n}$. From here it is clear that if $\lambda \in \mathbb{Z}^{n}$ and $s \cdot \lambda \in S_{Q_{n}}$ for some $s \in \mathbb{N}$, then $\lambda \in S_{Q_{n}}$. Hence, $Q_{n}$ is normal.

A convenient consequence of normality is that every lattice point in the $t$-th dilation of a normal polytope $P$ can be written as a sum of $t$, not necessarily distinct, lattice points in $P$. With that, we can prove the second part of our main theorem.

Theorem 4.16 (Main Theorem, Part 2). For every finitely generated, graded $S$ module $M$ with a 2-linear minimal free resolution and $\beta_{0,0}(M)=m$, there is a collection of $m$ threshold graphs $\left\{T_{1}, \ldots, T_{m}\right\}$, not necessarily distinct, such that $\beta(M)=\beta\left(\mathbb{k}\left[T_{1}\right] \oplus \cdots \oplus \mathbb{k}\left[T_{m}\right]\right)$.

Proof. By Theorem 3.2, $\omega(M)$ is a lattice point in $m P_{n}$ and is a sum of $m$ lattice points $p_{1}, \ldots, p_{m}$ in $P_{n}$, by Proposition 4.15. Applying Theorem 4.12 yields a threshold graph $T_{i}$ such that $p_{i}=\omega\left(\mathbb{k}\left[T_{i}\right]\right)$ for each $i \in[m]$, and thus,

$$
\beta(M)=\beta\left(T_{1}\right)+\cdots+\beta\left(T_{m}\right)=\beta\left(\mathbb{k}\left[T_{1}\right] \oplus \cdots \oplus \mathbb{k}\left[T_{m}\right]\right) .
$$

Remark 4.17. The decomposition in Theorem 4.16 is often not unique. So in the more general setting of modules, we do not know how to construct the family of trees representing a given Betti diagram as we do in the special case of algebras, see Theorem 4.12 and Example 4.14.

\section{The geometry of $P_{n}$ and $Q_{n}$}

In the previous section, we used the geometry of the lattice simplex $P_{n}$ of reduced Betti vectors of 2-linear ideals in $S$ (or equivalently, the lattice simplex $Q_{n}$ of nonzero anti-lecture-hall compositions of length $n$ ) to prove algebraic statements about Betti diagrams of algebras and modules with 2-linear resolutions, but these polytopes have many other beautiful geometric properties which make them interesting on their own. In this section, we take the opportunity to showcase a few of these properties. Specifically, we remark that $P_{n}$ has a simple Ehrhart polynomial, by a result from [Corteel et al. 2005], and we prove that $P_{n}$ is reflexive.

Given a $d$-dimensional polytope $P$, let $\operatorname{Ehr}_{P}(t)$ denote the number of lattice points in $t P$. It is well known that $\operatorname{Ehr}_{P}(t)$ is a degree $d$ polynomial in $t$, called the Ehrhart polynomial of $P$, with constant term 1 and leading coefficient equal to the volume of $P$, and that Ehrhart polynomials are preserved under unimodular transformations. For an introduction to Ehrhart theory, see [Beck and Robins 2007]. 
Theorem 5.1. For every $n, t \in \mathbb{N}, \operatorname{Ehr}_{P_{n}}(t)=\operatorname{Ehr}_{Q_{n}}(t)=(t+1)^{n}-t^{n}$.

Proof. Since the matrix $\Psi^{-1}$ in the proof of Proposition 4.11 is unimodular, we know that $\operatorname{Ehr}_{P_{n}}(t)=\operatorname{Ehr}_{Q_{n}}(t)$. So, let $A_{n}(t)$ denote the number of anti-lecture-hall compositions of length $n$ with $\lambda_{1} \leq t$. Theorem 4.10 gives us $A_{n}(t)=(t+1)^{n}$. Since every point in the $t Q_{n}$ satisfies $\lambda_{1}=t$, it follows immediately that

$$
\operatorname{Ehr}_{P_{n}}(t)=\operatorname{Ehr}_{Q_{n}}(t)=A_{n}(t)-A_{n}(t-1)=(t+1)^{n}-t^{n} .
$$

Next, we prove that $P_{n}$ is reflexive. For this, we need the concept of a dual (or polar) of a polytope, but restrict to the case of simplices, since those are the only polytopes we consider.

Definition 5.2. Let the vertices of a $d$-simplex $P$ be recorded as the rows of the $d \times(d-1)$ matrix $M$ and let $M^{*}$ be the $(d-1) \times d$ matrix such that $M M^{*}$ has value -1 everywhere outside the diagonal. The $d$-simplex whose vertices are the columns of $M^{*}$ is the dual $P^{*}$ of $P$.

If $P$ is a lattice polytope containing 0 as an interior point such that $P^{*}$ is also a lattice polytope, then $P$ and $P^{*}$ are called reflexive. These polytopes have several interesting properties and characterizations, for instance, a lattice polytope $P$ is reflexive if and only if its only interior lattice point is 0 and if $u$ and $v$ are two lattice points on the boundary of $P$, then either $u$ and $v$ are on the same facet, or $u+v$ is in $P$. This is an important concept with interesting connections to geometry and theoretical physics. For an exposition suitable for researchers with a background in discrete mathematics, we refer to Batyrev and Nill [2008].

Because $P_{n}$ is an $(n-1)$-dimensional simplex with coordinates in $\mathbb{Z}^{n}$, for each lattice point $p \in P_{n}$, we define

$$
p_{t}=\left[p_{1}, \ldots, p_{n-1}\right]:=\left[p-\eta_{n}\right]_{2 \leq i \leq n}=\left[p_{2}-\left(\begin{array}{l}
n \\
2
\end{array}\right), \ldots, p_{n}-\left(\begin{array}{l}
n \\
n
\end{array}\right)\right]
$$

to be the truncated coordinates of $p$ in $P_{n}$.

Theorem 5.3. The simplex $P_{n}$ realized in the truncated coordinates is a reflexive lattice polytope.

Proof. We begin by removing the left-most column of $\Omega$ to get the $n \times(n-1)$ matrix $\Omega_{n}^{\prime}$. Then the truncated coordinates of $P_{n}$ are the rows of $\Omega_{n}=\Omega_{n}^{\prime}-\eta_{n} \mathbf{1}_{n}$. More explicitly, the $i j$-entry of $\Omega_{n}^{\prime}$ is $(j+1)\left(\begin{array}{c}i+1 \\ j+2\end{array}\right)$ and the $j$ entry of $\eta_{n}$ is $\left(\begin{array}{c}n \\ j+1\end{array}\right)$.

The dual of $P_{n}$, in truncated coordinates, is the simplex whose vertices are the columns of the $(n-1) \times(n)$ matrix $\Xi_{n}$ satisfying that all values of $\Omega_{n} \Xi_{n}$ outside the diagonal are -1 . If all entries of $\Xi_{n}$ are integers, then the dual of $P_{n}$ is a lattice polytope and hence, $P_{n}$ is reflexive. To show this, we construct $\Xi_{n}$ explicitly with three $(n-1) \times n$ matrices, $\Xi_{n}^{\prime}, \Xi_{n}^{\prime \prime}$, and $\Xi_{n}^{\prime \prime \prime}$. The $i j$-entries of $\Xi_{n}^{\prime}$ are $-(i+2)(-1)^{i+j}\left(\begin{array}{c}i \\ j-1\end{array}\right)$ and the matrices $\Xi_{n}^{\prime \prime}$ and $\Xi_{n}^{\prime \prime \prime}$ are all zero, with the exceptions 
that the first column of $\Xi_{n}^{\prime \prime}$ is $-2(-1)^{i}$, and the bottom right-most entry of $\Xi_{n}^{\prime \prime \prime}$ is $1-n$. We consider $\Xi_{n}=\Xi_{n}^{\prime}+\Xi_{n}^{\prime \prime}+\Xi_{n}^{\prime \prime \prime}$.

To calculate the product $\Omega_{n} \Xi_{n}$, we separate both $\Omega_{n}$ and $\Xi_{n}$ into the sums above and then multiply them. The matrix multiplications are straightforward applications of elementary combinatorics, so we only record the results:

(1) The matrix $\Omega_{n}^{\prime} \Xi_{n}^{\prime}$ is the sum of two matrices. The only nonzero elements of the first are the diagonal $i i$-entries $i(i+1)$ and the only nonzero elements of the second are the first column $i 1$-entries $-i(i+1)$.

(2) The matrix $\eta_{n} \mathbf{1}_{n} \Xi_{n}^{\prime}$ has $1 \mathrm{~s}$ everywhere, except that the first column is constant with $-2 n+1$ and the last column is $n+1$.

(3) The matrix $\Omega_{n}^{\prime} \Xi_{n}^{\prime \prime}$ has 0 s everywhere, except that the first column's $i 1$-entry is $i(i+1)-2$.

(4) The matrix $\eta_{n} \mathbf{1}_{n} \boldsymbol{\Xi}_{n}^{\prime \prime}$ has 0 s everywhere, except that the first column is constant with $2 n-2$.

(5) The matrix $\Omega_{n}^{\prime} \Xi_{n}^{\prime \prime \prime}$ has 0 s everywhere, except that the rightmost bottom corner is $-n^{2}$.

(6) The matrix $\eta_{n} \mathbf{1}_{n} \mathbf{\Xi}_{n}^{\prime \prime \prime}$ has 0 s everywhere, except that the rightmost column is constant $-n$.

Summing up, we conclude that the $i j$-entry of $\Omega_{n} \Xi_{n}=\left(\Omega_{n}^{\prime}-\eta_{n} \mathbf{1}_{n}\right)\left(\Xi_{n}^{\prime}+\Xi_{n}^{\prime \prime}+\Xi_{n}^{\prime \prime \prime}\right)$ is

$$
-1 \text { if } i \neq j, \quad i^{2}+i-1 \text { if } i=j<n, \quad n \text { if } i=j=n .
$$

\section{Acknowledgements}

The authors thank Mats Boij, for several insightful conversations regarding this work, Benjamin Braun, for pointing out the connection to anti-lecture-hall compositions, and the anonymous referee, for the helpful suggestions to improve this paper. Engström thanks the Miller Institute for Basic Research at UC Berkeley for funding. Stamps thanks the Mathematical Sciences Research Institute for support to attend the 2011 Summer Graduate Workshop on Commutative Algebra.

\section{References}

[Barvinok 2002] A. Barvinok, A course in convexity, Graduate Studies in Mathematics 54, American Mathematical Society, Providence, RI, 2002. MR 2003j:52001 Zbl 1014.52001

[Batyrev and Nill 2008] V. Batyrev and B. Nill, "Combinatorial aspects of mirror symmetry", pp. 35-66 in Integer points in polyhedra-geometry, number theory, representation theory, algebra, optimization, statistics (Snowbird, UT, 2006), edited by M. Beck et al., Contemp. Math. 452, Amer. Math. Soc., Providence, RI, 2008. MR 2009m:14059 Zbl 1161.14037 
[Beck and Robins 2007] M. Beck and S. Robins, Computing the continuous discretely: Integer-point enumeration in polyhedra, Springer, New York, 2007. MR 2007h:11119 Zbl 1114.52013

[Boij and Söderberg 2008] M. Boij and J. Söderberg, "Graded Betti numbers of Cohen-Macaulay modules and the multiplicity conjecture", J. Lond. Math. Soc. (2) 78:1 (2008), 85-106. MR 2009g: 13018 Zbl 1189.13008

[Boij and Söderberg 2012] M. Boij and J. Söderberg, "Betti numbers of graded modules and the multiplicity conjecture in the non-Cohen-Macaulay case", Algebra Number Theory 6:3 (2012), 437-454. MR 2966705 Zbl 1259.13009

[Bousquet-Mélou and Eriksson 1997a] M. Bousquet-Mélou and K. Eriksson, "Lecture hall partitions", Ramanujan J. 1:1 (1997), 101-111. MR 99c:05015 Zbl 0909.05008

[Bousquet-Mélou and Eriksson 1997b] M. Bousquet-Mélou and K. Eriksson, "Lecture hall partitions, II”, Ramanujan J. 1:2 (1997), 165-185. MR 99c:05016 Zbl 0909.05009

[Bruns et al. 1997] W. Bruns, J. Gubeladze, and N. V. Trung, "Normal polytopes, triangulations, and Koszul algebras”, J. Reine Angew. Math. 485 (1997), 123-160. MR 99c:52016 Zbl 0866.20050

[Corteel and Savage 2003] S. Corteel and C. D. Savage, "Anti-lecture hall compositions", Discrete Math. 263:1-3 (2003), 275-280. MR 2003m:05007 Zbl 1019.05004

[Corteel et al. 2005] S. Corteel, S. Lee, and C. D. Savage, "Enumeration of sequences constrained by the ratio of consecutive parts", Sém. Lothar. Combin. 54A (2005), [article] B54Aa. MR 2006f:05011 Zbl 1086.05010

[Diestel 2010] R. Diestel, Graph theory, 4th ed., Graduate Texts in Mathematics 173, Springer, Heidelberg, 2010. MR 2011m:05002 Zbl 1204.05001

[Dochtermann and Engström 2009] A. Dochtermann and A. Engström, "Algebraic properties of edge ideals via combinatorial topology”, Electron. J. Combin. 16:2 (2009), 1-24. MR 2010f:13027 Zbl 1161.13013

[Eisenbud and Schreyer 2009] D. Eisenbud and F.-O. Schreyer, "Betti numbers of graded modules and cohomology of vector bundles", J. Amer. Math. Soc. 22:3 (2009), 859-888. MR 2011a:13024 Zbl 1213.13032

[Fløystad 2012] G. Fløystad, "Boij-Söderberg theory: Introduction and survey", pp. 1-54 in Progress in commutative algebra, I, edited by C. Francisco et al., de Gruyter, Berlin, 2012. MR 2932580 Zbl 1260.13020

[Fröberg 1990] R. Fröberg, “On Stanley-Reisner rings”, pp. 57-70 in Topics in algebra, II (Warsaw, 1988), edited by S. Balcerzyk et al., Banach Center Publ. 26, PWN, Warsaw, 1990. MR 93f:13009 Zbl 0741.13006

[Goodarzi and Yassemi 2012] A. Goodarzi and S. Yassemi, "Shellable quasi-forests and their $h-$ triangles”, Manuscripta Math. 137:3-4 (2012), 475-481. MR 2012m:13041 Zbl 1246.13029

[Herzog et al. 2012] J. Herzog, L. Sharifan, and M. Varbaro, "Graded Betti numbers of componentwise linear ideals", preprint, 2012. To appear in Proc. Amer. Math. Soc. arXiv 1111.0442

[Klivans 2007] C. J. Klivans, "Threshold graphs, shifted complexes, and graphical complexes", Discrete Math. 307:21 (2007), 2591-2597. MR 2008j:05373 Zbl 1127.05086

[Mahadev and Peled 1995] N. V. R. Mahadev and U. N. Peled, Threshold graphs and related topics, Annals of Discrete Mathematics 56, North-Holland, Amsterdam, 1995. MR 97h:05001 Zbl 0852.05001

[Nagel and Sturgeon 2013] U. Nagel and S. Sturgeon, "Combinatorial interpretations of some BoijSöderberg decompositions”, J. Algebra 381 (2013), 54-72. MR 3030509 
[Woodroofe 2011] R. Woodroofe, "Erdős-Ko-Rado theorems for simplicial complexes", J. Combin. Theory Ser. A 118:4 (2011), 1218-1227. MR 2012a:05351 Zbl 1231.05308

Communicated by David Eisenbud

Received 2012-11-06 Revised 2013-01-25 Accepted 2013-03-12

alexander.engstrom@aalto.fi Department of Mathematics, Aalto University, P.O. Box 11100, Fl-00076 Aalto, Finland

matthew.stamps@aalto.fi_ Department of Mathematics, Aalto University,

P.O. Box 11100, Fl-00076 Aalto, Finland 


\section{Algebra \& Number Theory}

msp.org/ant

\section{EDITORS}

MANAGING EDITOR

Bjorn Poonen

Massachusetts Institute of Technology

Cambridge, USA

\author{
EDITORIAL BOARD CHAIR \\ David Eisenbud \\ University of California \\ Berkeley, USA
}

\section{BOARD OF EDITORS}

Georgia Benkart

Dave Benson

Richard E. Borcherds

John H. Coates

J-L. Colliot-Thélène

Brian D. Conrad

Hélène Esnault

Hubert Flenner

Edward Frenkel

Andrew Granville

Joseph Gubeladze

Roger Heath-Brown

Ehud Hrushovski

Craig Huneke

Mikhail Kapranov

Yujiro Kawamata

János Kollár

Yuri Manin

Barry Mazur

Philippe Michel
University of Wisconsin, Madison, USA

University of Aberdeen, Scotland

University of California, Berkeley, USA

University of Cambridge, UK

CNRS, Université Paris-Sud, France

University of Michigan, USA

Freie Universität Berlin, Germany

Ruhr-Universität, Germany

University of California, Berkeley, USA

Université de Montréal, Canada

San Francisco State University, USA

Oxford University, UK

Hebrew University, Israel

University of Virginia, USA

Yale University, USA

University of Tokyo, Japan

Princeton University, USA

Northwestern University, USA

Harvard University, USA

École Polytechnique Fédérale de Lausanne
Susan Montgomery

Shigefumi Mori

Raman Parimala

Jonathan Pila

Victor Reiner

Karl Rubin

Peter Sarnak

Joseph H. Silverman

Michael Singer

Vasudevan Srinivas

J. Toby Stafford

Bernd Sturmfels

Richard Taylor

Ravi Vakil

Michel van den Bergh

Marie-France Vignéras

Kei-Ichi Watanabe

Efim Zelmanov

Shou-Wu Zhang
University of Southern California, USA

RIMS, Kyoto University, Japan

Emory University, USA

University of Oxford, UK

University of Minnesota, USA

University of California, Irvine, USA

Princeton University, USA

Brown University, USA

North Carolina State University, USA

Tata Inst. of Fund. Research, India

University of Michigan, USA

University of California, Berkeley, USA

Harvard University, USA

Stanford University, USA

Hasselt University, Belgium

Université Paris VII, France

Nihon University, Japan

University of California, San Diego, USA

Princeton University, USA

PRODUCTION

production@msp.org

Silvio Levy, Scientific Editor

See inside back cover or msp.org/ant for submission instructions.

The subscription price for 2013 is US $\$ 200 /$ year for the electronic version, and $\$ 350 /$ year $(+\$ 40$, if shipping outside the US) for print and electronic. Subscriptions, requests for back issues and changes of subscribers address should be sent to MSP.

Algebra \& Number Theory (ISSN 1944-7833 electronic, 1937-0652 printed) at Mathematical Sciences Publishers, 798 Evans Hall \#3840, c/o University of California, Berkeley, CA 94720-3840 is published continuously online. Periodical rate postage paid at Berkeley, CA 94704, and additional mailing offices.

ANT peer review and production are managed by EditFLOW ${ }^{\circledR}$ from Mathematical Sciences Publishers.

\section{PUBLISHED BY}

- mathematical sciences publishers

nonprofit scientific publishing

http://msp.org/

(C) 2013 Mathematical Sciences Publishers 


\section{Algebra \& Number Theory}

$\begin{array}{lll}\text { Volume } 7 & \text { No. } 7 \quad 2013\end{array}$

Weil representation and transfer factor

TERUJI THOMAS

Analytic families of finite-slope Selmer groups

1571

JONATHAN POTTHARST

Multiplicative excellent families of elliptic surfaces of type $E_{7}$ or $E_{8}$

ABHINAV KUMAR and TETSUJI SHIODA

Cohomological invariants of algebraic tori

SAM Blinstein and AleXANDer MerkurJeV

On abstract representations of the groups of rational points of algebraic groups and their 1685 deformations

IGOR A. RAPINCHUK

Betti diagrams from graphs

AlEXANDER ENGSTRÖM and MATTHEW T. STAMPS

Hopf monoids from class functions on unitriangular matrices

Marcelo Aguiar, NANTEl Bergeron and Nathaniel Thiem 